\title{
Sleep habits and sleep disturbances in Dutch children: a population-based study
}

\author{
Raphaële Reine Lydie van Litsenburg • \\ Ruth C. Waumans • Gerrit van den Berg • \\ Reinoud J. B. J. Gemke
}

Received: 11 January 2010 /Accepted: 11 February 2010/Published online: 1 March 2010

(C) The Author(s) 2010. This article is published with open access at Springerlink.com

\begin{abstract}
Sleep disorders can lead to significant morbidity. Information on sleep in healthy children is necessary to evaluate sleep disorders in clinical practice, but data from different societies cannot be simply generalized. The aims of this study were to (1) assess the prevalence of sleep disturbances in Dutch healthy children, (2) describe sleep habits and problems in this population, (3) collect Dutch norm data for future reference, and (4) compare sleep in children from different cultural backgrounds. A populationbased descriptive study was conducted using the Children's sleep habits questionnaire and the sleep self-report. One thousand five hundred seven proxy-reports and 262 selfreports were analyzed. Mean age was 8.5 years $(95 \%$ confidence interval, 8.4-8.6), 52\% were boys. Sleep problems in Dutch children were present in $25 \%$, i.e., comparable to other populations. Sleep habits were age-related. Problem sleepers scored significantly higher on all scales. Correlations between parental and self-assessments were low to moderate. Dutch children had significantly more sleep disturbances than children from the USA and less than Chinese children. Cognitions and attitudes towards what is considered normal sleep seem to affect the appraisal of sleep, this probably accounts partly for cultural differences. For a better understanding of cultural influences on sleep, more information on these determinants and the establishment of cultural norms are mandatory.
\end{abstract}

R. R. L. van Litsenburg $(\varangle) \cdot$ R. C. Waumans $\cdot$ G. van den Berg • R. J. B. J. Gemke

Department of Pediatrics, VU University Medical Center,

9D11 secretariaat kindergeneeskunde, P.O. Box 7057, 1007 MB

Amsterdam, Netherlands

e-mail: Litsenburg@vumc.nl
Keywords Sleep · Children's sleep habits questionnaire (CSHQ) - Sleep self-report (SSR) · Cultural comparison . Dutch

\section{Introduction}

Sleep disorders in children can lead to significant medical and behavioral morbidity. Previous studies have shown a prevalence of sleep disorders in children up to $30 \%[2,3$, 17]. Some sleep problems are more common during certain stages of child development, such as night wakings during infancy [28], Children with sleep difficulties experience higher rates of behavioral problems, depression, anxiety in adulthood, impaired cognitive function, learning disabilities, and emotional development [1, 7, 9, 10, 15, 27]. Sleep problems are more common in certain medical conditions, such as chronic pain, attention deficit hyperactivity disorder (ADHD), and autism [16, 19, 22].

In order to adequately evaluate sleep difficulties in clinical practice, information on sleep in healthy children is essential. Relatively few studies have addressed sleep habits in healthy school-aged children, and there are no recent studies assessing sleep in Dutch school-aged children. Sleep is influenced by many different determinants such as biological and psychological factors, child characteristics and development, and social and environmental surroundings. Cultural differences also play an important role in sleep habits and perceived problems [14, 17, 20, 21]. Cultural norms determine the boundaries between "normal" and "problematic" sleep behavior, so data on sleep habits and problems cannot simply be generalized [13]. Studies that have evaluated sleep in children have used a variety of instruments and measures, complicating direct comparison. To enable intercultural 
comparison of pediatric sleep problems, valid, reliable, and international accepted instruments should be used.

The aims of this study were to (1) assess the prevalence of sleep disturbances in Dutch healthy children, (2) describe sleep habits and problems in this population, (3) collect Dutch norm data for future reference, and (4) compare sleep in children from different cultural backgrounds with reliable, valid, and generalizable measures. Therefore, the children's sleep habits questionnaire (CSHQ) and the sleep self-report (SSR) were translated to Dutch. These questionnaires were developed in the USA as screening instruments for sleep habits and problems in school-aged children. The CSHQ has shown adequate reliability and validity [24] and has been used in several population-based studies. With permission of the original author, the CSHQ and SSR were translated into Dutch, including two independent forward translations and an independent backward translation. Any discrepancies were solved by consensus discussions.

\section{Methods}

\section{Study design}

The study was conducted in 14 randomly chosen daycare centers and elementary schools in urban and suburban areas, between December 2006 and April 2007. The CSHQ was distributed to all parents of children over 2 years of age with written information about the study and a stamped return envelope. To enhance participation, the study was announced through internal school magazines, and written reminders were posted throughout the schools whenever possible. Parents were informed of the voluntary character of the study. Information on demographic variables, sleeping conditions, and family situation was collected additionally. In order to assess parent-child agreement, the SSR was distributed in randomly chosen schools to children between the ages of 7 and 12. Most questionnaires were self-administrated, except for the youngest classes in which the children were assisted by the teacher and/or research assistant. Identical case numbers linked parental and self-reports. The study was approved by our Institutional Review Board.

\section{Measures}

The abbreviated version of the CSHQ and the SSR were used to assess sleep habits and disturbances. The CSHQ is a 1-week recall, 33-item parental questionnaire that was developed as a sleep screening tool for school-aged children [24]. It has been used in several studies [4-6, 17, 22-24] and has adequate psychometric properties in children living in the USA [24]. Recently, the CSHQ has been shown to be a useful screening tool in younger children as well [8]. In our study, Cronbach's $\alpha$ coefficients ranged from 0.47 to 0.68 , comparable to the original CSHQ. Parents rated the frequency of sleep behavior for the most recent "typical" week on a three-point Likert scale, with the response options usually (five to seven times per week), sometimes (two to four times per week), and rarely (zero to one time per week). A higher score indicates more sleep disturbances. Also, parents were asked to indicate whether a specific sleep item is a problem by circling yes, no, or not applicable for each item. Information on habitual bedtime, morning wake-up time, and sleep duration was collected additionally. The CSHQ allows for a total score over 33 items and subscales scores on a number of key sleep domains: bedtime resistance (six items), sleep onset delay (one item), sleep duration (three items), sleep anxiety (four items), night wakening (three items), parasomnias (seven items), sleep-disordered breathing (three items), and daytime sleepiness (eight items). See Table 1 for clarification of the sleep disorders.

The SSR is a 26-item, 1-week recall questionnaire designed for interviewing or self-administration by children from 7 to 12 years of age $[22,23]$. It comprises a three-point Likert scale, with the same response options as the CSHQ. Higher scores indicate more disturbed sleep. The SSR allows for a total score based on 13 items, directly relating to the same sleep domains as incorporated in the CSHQ [22]. Comparison of the CSHQ and the SSR is possible for specific items.

\section{Statistical analysis}

The Statistical Package for Social Sciences for Windows version 15.0 was used for all data analyses. For the description of questionnaire scores, means and 95\% confidence intervals (CI) were calculated. $t$ Tests for independent samples and Mann-Whitney $U$ tests were used to assess between-group differences on total and subscale scores. The effect of age, class, and living situation (living with both parents, single-parent families, alternatively with mother or father) was assessed using linear regression analysis and analysis of variance. Spearman correlations were calculated to examine correlations between CSHQand SSR-related items. Separate analyses were performed for problem sleepers, which were defined as children who had at least one sleep item endorsed as a problem. Significance level was set at $p<0.05$ for all analyses.

\section{Results}

Demographics

Two thousand four hundred fifty-three questionnaires were distributed. Information on 1,552 children was returned 
Table 1 Sleep disorder descriptions

\section{Description}

Bedtime resistance

This category assesses if the child falls asleep in his/her own bed, if he/she has a regular bedtime, if he/she needs a parent in the room, struggles at bedtime, or is afraid of sleeping alone

Sleep onset delay Is defined as a delay of $20 \mathrm{~min}$ or more

Sleep duration Assesses if the sleep duration is adequate and whether the child sleeps the same amount each day

Sleep anxiety

Night wakening Does the child need a parent in the room, is he/she afraid of sleeping alone or in the dark, does he/she have trouble sleeping away from home?

Most healthy young children wake up during the night but usually resume sleep without parental awareness or intervention. Referred children are more likely to have difficulty falling back to sleep and require more parental interventions. Questions include frequency of night wakenings and switching to another person's bed [28]

Parasomnias

Sleep-disordered breathing

Sleep disorders due to central nervous system arousal, leading to motor or autonomic activation [2]. Assesses bedwetting, talking during sleep, sleepwalking, bruxism, night terrors, and restlessness

This category includes primary snoring, upper airway resistance syndrome, obstructive hypoventilation, and obstructive sleep apnea. It is most prevalent in children 2-6 years old (due to adenotonsillar hypertrophy), in adolescents (related to obesity), and during the first year of life (anatomic and neurologic problems) [28]

Daytime sleepiness This category includes questions regarding waking up in the morning (with help from others, time to alertness, difficulties getting up, and negative mood) and sleepiness during the day (falling asleep watching TV or riding in a car)

(response rate 63\%); no information was available on nonrespondents. Most children (94\%) had no comorbidities, did not use any medication (93\%), and were born in the Netherlands (99\%). Most reported medical problems were ADHD $(n=26)$, autism $(n=8)$, and physical problems $(n=25)$. The most commonly used medication was for asthma and allergies, followed by drugs for ADHD. All children reported to have ADHD or autism were excluded from analysis. Two other children treated with herbal medicine against sleep problems were also excluded. Five questionnaires were not filled out correctly, and another four children had not yet reached the age of 2 ; they were excluded as well. The final number of analyzed questionnaires was 1,507 . Respondents were mostly mothers $(87 \%)$. Mean child age was 8.5 years $(95 \%$ CI, 8.4-8.6 ; range, $2.0-$ 13.7), $52 \%$ were boys. Ten percent of all children shared their bedroom with a sibling, $0.8 \%$ with a parent.

\section{Children's sleep habits questionnaire}

Mean bedtime was 8:00 p.m. (range, 6:20-10:30 p.m.), and mean wake-up time was 7:06 a.m. (range, 4:00-9:15 a.m.). Sleep schedules were age-related: bedtime and wake-up time were later for older children $(p<.001)$. Mean sleep duration was $10.66 \mathrm{~h}$ (95\% CI, 10.57-10.75; range, 7.00$14.5 \mathrm{~h})$ and decreased significantly with child age $(p<.001)$. Mean awake minutes at night was 7.89 (95\% CI, 7.148.63; range, 1-60 $\mathrm{min})$, this was not age-related.

CSHQ scores for all children and per age range (2-6, 710 , and 10-14 years) are summarized in Table 2. Mean CSHQ total score was 40.50 (95\% CI, 40.21-40.80) and was not influenced by age. Older children scored higher on sleep onset delay $(p<.001)$, sleep duration $(p=.02)$, and daytime sleepiness $(p<.001)$, indicating more problems in these areas. Younger children had more problems on the subscales of bedtime resistance $(p<.01)$, sleep anxiety $(p<.001)$, night wakenings $(p<.04)$, parasomnias $(p<.001)$, and sleep-disordered breathing $(p=.014)$. Girls had more disturbances than boys, reflected in total score (boys: mean, 40.15; 95\% CI, 39.74-40.56; girls: mean, 40.89; 95\% CI, 40.46-41.32; $p=.014)$, sleep onset delay (boys: mean, 1.25; 95\% CI, 1.21-1.29; girls: mean, 1.34; 95\% CI, $1.30-1.39 ; p=.002$ ), and daytime sleepiness (boys: mean, 10.75; 95\% CI, 10.57-10.92; girls: mean, 11.60; 95\% CI, 11.38-11.82; $p<.001)$. Children sharing a bedroom scored significantly higher on bedtime resistance $(p<.001)$. Children living in single-parent families had a higher total score than children in a two-parent household (mean, 41.77 and 40.35, respectively, $p<0.03$ ). Children from single-parent families also showed more problems on the bedtime resistance (mean, 7.05 versus $6.64 ; p<0.03$ ), anxiety (mean, 5.19 versus $4.83 ; p=0.02$ ), and night wakening (mean, 3.86 versus $3.59 ; p<.04$ ) subscales. Separate analyses for comorbidity or the use of prescription medication were not performed because these subgroups were small and heterogeneous.

Parents of $383(25 \%)$ children endorsed at least one item out of 33 of the CSHQ as a problem; these children scored significantly higher on all scales (see Table 3 ). One hundred fifty-two (10\%) children had at least one problem endorsed for daytime sleepiness, 138 (9\%) for sleep duration, 132 (9\%) for bedtime resistance, 112 (7\%) for parasomnias, 106 (7\%) for sleep anxiety, 83 (6\%) for sleep onset delay, 45 (3\%) for night wakening, and 16 (1\%) for sleep-disordered 
Table 2 Children's sleep habits questionnaire scores for all children and per age group

\begin{tabular}{|c|c|c|c|c|c|c|c|c|}
\hline \multirow[t]{2}{*}{ CSHQ score } & \multicolumn{2}{|l|}{ All } & \multicolumn{2}{|l|}{ 2-6years } & \multicolumn{2}{|l|}{ 7-10years } & \multicolumn{2}{|l|}{ 10-14years } \\
\hline & Mean $(95 \% \mathrm{CI})$ & $N$ & Mean $(95 \% \mathrm{CI})$ & $N$ & Mean $(95 \% \mathrm{CI})$ & $N$ & Mean $(95 \% \mathrm{CI})$ & $N$ \\
\hline Total score & $40.50(40.21-40.80)$ & 1,282 & $40.83(40.21-41.44)$ & 283 & $40.57(40.09-41.04)$ & 554 & $40.16(39.66-40.65)$ & 412 \\
\hline \multicolumn{9}{|l|}{ Subscale item } \\
\hline Bedtime resistance ${ }^{\mathrm{b}}$ & $6.68(6.61-6.76)$ & 1,375 & $6.83(6.64-7.02)$ & 305 & $6.74(6.62-6.86)$ & 583 & $6.48(6.38-6.59)$ & 452 \\
\hline Sleep onset delay ${ }^{\mathrm{a}}$ & $1.30(1.27-1.33)$ & 1,507 & $1.12(1.08-1.16)$ & 323 & $1.29(1.24-1.33)$ & 632 & $1.43(1.37-1.49)$ & 507 \\
\hline Sleep duration ${ }^{\mathrm{a}}$ & $3.50(3.45-3.55)$ & 1,430 & $3.39(3.30-3.48)$ & 310 & $3.49(3.42-3.57)$ & 606 & $3.57(3.48-3.66)$ & 478 \\
\hline Sleep anxiety ${ }^{\mathrm{b}}$ & $4.86(4.79-4.93)$ & 1,359 & $5.10(4.94-5.26)$ & 300 & $4.96(4.84-5.07)$ & 580 & $4.56(4.46-4.67)$ & 445 \\
\hline Night wakening ${ }^{\mathrm{b}}$ & $3.62(3.56-3.67)$ & 1,368 & $3.91(3.76-4.05)$ & 304 & $3.59(3.50-3.67)$ & 583 & $3.44(3.37-3.52)$ & 443 \\
\hline Parasomnias $^{\mathrm{b}}$ & $8.57(8.48-8.66)$ & 1,355 & 8.89 (8.68-9.09) & 301 & $8.64(8.50-8.78)$ & 584 & $8.23(8.10-8.36)$ & 437 \\
\hline Sleep-disordered breathing ${ }^{\mathrm{b}}$ & $3.30(3.26-3.33)$ & 1,318 & $3.40(3.30-3.49)$ & 293 & $3.29(3.24-3.35)$ & 567 & $3.24(3.19-3.29)$ & 422 \\
\hline Daytime sleepiness $^{\mathrm{a}}$ & $11.16(11.02-11.30)$ & 1,424 & $10.89(10.63-11.15)$ & 308 & $11.02(10.80-11.24)$ & 606 & $11.52(11.27-11.77)$ & 471 \\
\hline
\end{tabular}

$N=$ number of children included per (sub)scale. Number vary due to missing responses; scores were calculated if less than $50 \%$ of items were missing

${ }^{\text {a }}$ Significantly higher score (more problems) in older children

${ }^{\mathrm{b}}$ Significantly higher score (more problems) in younger children

breathing. There were no significant differences in age and gender between problem sleepers and non-problem sleepers. Problem sleepers scored significantly higher on all subscales and on the total score (see Table 3).

\section{Sleep self-report}

Two hundred seventy children filled in the sleep self-report. Three children diagnosed with ADHD and five with autism were omitted from analyses; 262 questionnaires were included for the final analysis. Mean age was 10.34 years (95\% CI, 10.18-10.50; range, $8-12$ ), 47\% were boys. Of all children, $10.3 \%$ attended class $4,10.3 \%$ attended class 5 , $11.8 \%$ attended class $6,33.2 \%$ attended class 7 , and $33.6 \%$ attended class 8 (i.e., the highest grade in elementary school). There were no significant differences between boys and girls. Children who indicated they had trouble sleeping scored significantly higher (mean total score, 18.35 versus $22.83 ; p<.001)$ on all SSR items. Children in the two lowest classes scored significantly higher than children in the three highest classes, $(p<.01)$, indicating more sleep problems in younger children. Mean total score over 13 items was 19.04 (95\% CI, 18.58-19.50).

Correlation children's sleep habits questionnaire and sleep self-report

For 155 (10\% of total sample) children, both a parent-report and a self-report were available. Spearman's correlations were calculated for all corresponding items (see Table 4). Correlations between parent and child answers were small to moderate in six out of the 11 corresponding items.
Table 3 Children's sleep habits questionnaire

*Problem sleepers versus nonproblem sleepers $p<.001$

${ }^{\text {a }}$ Problem sleepers were defined as children who had at least one item endorsed as a problem

\begin{tabular}{|c|c|c|c|c|}
\hline \multirow[t]{2}{*}{ CSHQ score } & \multicolumn{2}{|l|}{ Problem sleepers ${ }^{\mathrm{a}}$} & \multicolumn{2}{|l|}{ Non-problem sleepers } \\
\hline & Mean $(95 \% \mathrm{CI})$ & $N$ & Mean $(95 \% \mathrm{CI})$ & $N$ \\
\hline Total score* & $44.72(44.01-45.43)$ & 265 & $39.25(38.95-39.54)$ & 888 \\
\hline \multicolumn{5}{|l|}{ Subscale item } \\
\hline Bedtime resistance* & $8.81(8.29-9.34)$ & 85 & $6.54(6.48-6.61)$ & 1,290 \\
\hline Sleep onset delay* & $2.11(1.94-2.28)$ & 83 & $1.25(1.22-1.28)$ & 1,424 \\
\hline Sleep duration* & $4.88(4.56-5.20)$ & 78 & $3.42(3.37-3.46)$ & 1,352 \\
\hline Sleep anxiety* & $7.07(6.72-7.42)$ & 90 & $4.70(4.64-4.77)$ & 1,269 \\
\hline Night wakening* & $5.74(5.30-6.18)$ & 44 & $3.55(3.50-3.60)$ & 1,324 \\
\hline Parasomnias* & $10.85(10.42-11.28)$ & 93 & $8.40(8.32-8.49)$ & 1,262 \\
\hline Sleep-disordered breathing* & $5.08(4.40-5.75)$ & 13 & $3.28(3.24-3.31)$ & 1,305 \\
\hline Daytime sleepiness* & $13.77(13.20-14.34)$ & 112 & $10.94(10.80-11.07)$ & 1,312 \\
\hline
\end{tabular}


Table 4 Correlation between the sleep self-report and children's sleep habits questionnaire

\begin{tabular}{|c|c|c|c|c|}
\hline Item & SSR mean $(95 \% \mathrm{CI})$ & CSHQ mean $(95 \% \mathrm{CI})$ & $r^{\mathrm{a}}$ & $p$ \\
\hline Child falls asleep within $20 \mathrm{~min}$ & $2.11(1.99-2.23)$ & $1.39(1.28-1.49)$ & 0.42 & $<.001$ \\
\hline Child is afraid of sleeping alone & $1.16(1.09-1.23)$ & $1.12(1.06-1.18)$ & 0.35 & $<.001$ \\
\hline Child is afraid of sleeping in the dark & $1.35(1.26-1.45)$ & $1.30(1.20-1.41)$ & 0.24 & .005 \\
\hline Difficulty getting out of bed in the morning & $2.12(2.01-2.24)$ & $1.58(1.47-1.68)$ & 0.21 & .01 \\
\hline Child sleeps too little & $1.54(1.44-1.64)$ & $1.29(1.21-1.36)$ & 0.16 & NS \\
\hline Child falls asleep in parent's or sibling's bed & $1.33(1.23-1.43)$ & $1.12(1.06-1.18)$ & 0.16 & .05 \\
\hline Child moves to someone else's bed & $1.21(1.14-1.28)$ & $1.06(1.02-1.10)$ & 0.15 & .04 \\
\hline Child goes to bed at same time & $1.67(1.57-1.77)$ & $1.06(1.02-1.11)$ & 0.15 & NS \\
\hline Child seems tired & $1.56(1.47-1.66)$ & $1.30(1.22-1.39)$ & 0.06 & NS \\
\hline Child awakes once during night & $1.59(1.49-1.69)$ & $1.35(1.25-1.44)$ & 0.07 & NS \\
\hline Child struggles at bedtime & $1.34(1.25-1.42)$ & $1.13(1.05-1.20)$ & 0.05 & NS \\
\hline
\end{tabular}

SSR sleep self-report, $C S H Q$ children's sleep habits questionnaire, $N S$ not significant

${ }^{a}$ Spearman's correlation between mean SSR and CSHQ item scores

\section{Cultural comparison}

In order to compare sleep in Dutch children to sleep in children from different cultural backgrounds, the results from this study were compared with results found by Liu et al., who used the CSHQ in China and the USA [17]. The samples were comparable with regards to gender, living area, and social economic status, but there were seasonal differences. The US sample was recruited during winter, spring, and fall; the Chinese sample during fall; and our sample during winter and spring. To eliminate age effects, analyses were performed for age-matched samples.

In the US sample, a significantly lower CSHQ total score was found (mean, 38.71 \pm 5.51 ) indicating less sleep disturbances compared with Dutch children (mean, $40.67 \pm 5.50 ; p<.001)$. This was also the case for most subscale items, such as sleep anxiety, night wakening, parasomnias, and daytime sleepiness (all $p<.001$; see

Table 5 Cultural comparison between sleep in children from the Netherlands, USA, and China

\begin{tabular}{|c|c|c|c|c|c|c|c|c|c|c|c|c|}
\hline \multirow[t]{3}{*}{ CSHQ score } & \multicolumn{3}{|c|}{ Netherlands } & \multicolumn{3}{|l|}{$\mathrm{USA}^{\mathrm{a}}$} & \multicolumn{3}{|c|}{ Netherlands } & \multicolumn{3}{|c|}{ China $^{a}$} \\
\hline & \multicolumn{3}{|c|}{$\begin{array}{l}\text { Age } 7.53 \pm 1.98 \text { years } \\
\text { (range, } 4-11 \text { ) }\end{array}$} & \multicolumn{3}{|c|}{$\begin{array}{l}\text { Age } 7.56 \text { years } \\
\text { (range, } 4.8-11 \text { ) }\end{array}$} & \multicolumn{3}{|c|}{$\begin{array}{l}\text { Age } 10.07 \pm 1.78 \text { years } \\
\text { (range, } 7-13.7 \text { ) }\end{array}$} & \multicolumn{3}{|c|}{$\begin{array}{l}\text { Age 11years } \\
\text { (range, } 7-13 \text { ) }\end{array}$} \\
\hline & Mean & $\mathrm{SD}$ & $N$ & Mean & $\mathrm{SD}$ & $N$ & Mean & SD & $N$ & Mean & SD & $N$ \\
\hline Total score $e^{b, c}$ & 40.67 & 5.50 & 926 & 38.71 & 5.51 & 357 & 40.27 & 5.30 & 815 & 42.11 & 7.43 & 292 \\
\hline \multicolumn{13}{|l|}{ Subscale item } \\
\hline Bedtime resistance $\mathrm{e}^{\mathrm{b}, \mathrm{c}}$ & 6.71 & 1.45 & 987 & 7.01 & 1.80 & 393 & 6.60 & 1.29 & 878 & 7.92 & 2.49 & 292 \\
\hline Sleep onset delay ${ }^{\mathrm{c}}$ & 1.27 & 0.55 & 1,071 & 1.26 & 0.54 & 415 & 1.36 & 0.64 & 973 & 1.28 & 0.60 & 292 \\
\hline Sleep duration ${ }^{\mathrm{c}}$ & 3.49 & 0.93 & 1,020 & 3.44 & 0.98 & 408 & 3.54 & 0.97 & 925 & 4.21 & 1.53 & 292 \\
\hline Sleep anxiety ${ }^{\mathrm{c}, \mathrm{d}}$ & 4.97 & 1.38 & 979 & 4.86 & 1.43 & 387 & 4.74 & 1.25 & 868 & 5.58 & 1.98 & 292 \\
\hline Night wakening ${ }^{\mathrm{b}, \mathrm{c}}$ & 3.65 & 1.06 & 988 & 3.49 & 0.88 & 388 & 3.47 & 0.84 & 869 & 3.85 & 1.27 & 292 \\
\hline Parasomnias $^{\mathrm{b}}$ & 8.67 & 1.73 & 985 & 8.14 & 1.31 & 382 & 8.40 & 1.54 & 864 & 8.46 & 2.06 & 292 \\
\hline Sleep-disordered breathing ${ }^{\mathrm{c}}$ & 3.32 & 0.70 & 953 & 3.28 & 0.67 & 391 & 3.24 & 0.55 & 836 & 3.34 & 0.97 & 292 \\
\hline Daytime sleepiness ${ }^{\mathrm{b}, \mathrm{c}}$ & 11.06 & 2.65 & 1,019 & 9.72 & 2.89 & 387 & 11.27 & 2.83 & 915 & 10.22 & 3.10 & 292 \\
\hline
\end{tabular}

Dutch data was compared with data from Liu et al. [17]. The Dutch samples used in these analyses were selected to match the USA and China samples with regarding to age, in order to minimize any age effects

CSHQ children's sleep habits questionnaire

${ }^{\text {a }}$ Reference sample USA and China, Lui et al. [17]

${ }^{\mathrm{b}}$ Difference between Dutch and US children, $p<.001$

${ }^{\mathrm{c}}$ Difference between Dutch and Chinese children, $p<.001$

${ }^{\mathrm{d}}$ Difference between Dutch and US children, $p<.05$ 
Table 5) [17]. US children only scored higher for bedtime resistance $(p<.001)$. Chinese children scored significantly higher on the CSHQ score compared with Dutch children (mean, 42.11 \pm 7.43 and 40.27 \pm 5.30 , respectively; $p<.001$; see Table 3 ), indicating more sleep problems in this population [17]. Dutch children also scored significantly lower for bedtime resistance, sleep duration, sleep anxiety, night wakening, and sleepdisordered breathing (all $p<.001$ ). Dutch children did appear to have more problems with sleep onset delay and daytime sleepiness $(p<.001)$.

\section{Discussion}

\section{Dutch population}

The prevalence of sleep problems in healthy Dutch schoolaged children in this study was comparable to percentages found in other studies; $25 \%$ of all children were considered by their parents to have at least one sleep-related problem. Daytime sleepiness was reported most frequently as a problem (10\%), corresponding with other studies [10, 23]. Children marked as "problem sleepers" by either themselves or their parents had significantly higher scores. This is an interesting discriminating characteristic of the CSHQ and the SSR, which emphasizes the influence of cognitions and attitudes towards what is considered "normal" sleep.

Child age was associated with sleep duration, bedtime, and wake-up time. Specific sleep problems were more common in certain age groups, which might be a reflection of the child's normal development. For example, younger children had more problems with sleep anxiety and bedtime resistance, whereas for older children, problems with sleep onset delay and sleep duration were reported more often. Parents reported that girls experienced more sleep problems than boys, notably for sleep onset delay and daytime sleepiness, while for the child self-reports, no gender difference was found. Reports in the literature about age and gender effects are inconsistent [3, 23, 25].

Correlations between parental and self-assessment of child sleep were small to moderate. Owens et al. found no correlations at all in a healthy control group [22]. In general, questionnaire-based studies in pediatric populations have resulted in mixed outcomes regarding parental and self-assessment correlations [12, 30]. Although assessing children's sleep by means of parental questionnaires has shown adequate correlation with objective sleep measures (such as actigraphy) for sleep schedules, parents are less accurate in assessing sleep quality $[26,31,32]$, as certain aspects of sleep problems can be unnoticed by parents. Therefore, including child reports wherever possible seems preferable.
Cultural comparison

It is difficult to disentangle the separate influences of biology and culture on sleep habits and problems. Many different aspects may influence sleep, and these cannot always be accounted for in a population-based study. Using the CSHQ, we were able to compare collected data in Dutch children with available data from the US and Chinese children [17]. This demonstrated a similar prevalence of sleep disturbances [18] but also emphasized important differences. Overall, based on total CSHQ scores, US children experienced the least sleep problems, followed by Dutch and Chinese children, respectively. For some specific aspects of sleep, such as sleep onset delay, parasomnias, and daytime sleepiness, Dutch children had more problems as compared with both Chinese and US children. In contrast, Dutch children had fewer problems with bedtime resistance.

Although an in-depth investigation into the reasons of these cultural sleep differences is beyond the scope of this paper, some possible explanations deserve attention. Dutch cognitions and sleep traditions are probably more similar to US than Chinese practices, so many of the differences between the Chinese and Dutch data might be (partly) explained by reasons already mentioned by Liu et al. [17], e.g., the emphasis on individualism and independence versus collectivism and social harmony, the practice of cosleeping in Asian countries, and different school practices and requirements. A previous study into sleep patterns in US and Dutch children in 1996 showed Dutch children slept more than US children, which was thought to be related to the Dutch tradition of the three "R's": Rust, Reinheid, and Regelmaat (rest, cleanliness, and regularity) [29]. In this study, Dutch and US children had similar sleep duration. Higher scores on the CSHQ in Dutch children as compared with US children might reflect differences in child care practice and cognitions and attitudes towards normal sleep. The three "R's" with emphasis on rest and regularity might lead Dutch parents to expect a longer and more undisturbed sleep to be best for their children. Of course, this is an assumption because parental cognitions and attitudes were not included in this study.

\section{Limitations}

Possible limitations of this study include the response rate of $63 \%$. This might have caused bias, although it has been argued that there is little empirical support for bias as a direct result of low response rates [11]. Also, there was a remarkably low ADHD rate in our population (1.7\%), under-reporting of ADHD may have led to higher scores in the studied population, since sleep problems are more common in ADHD [22]. It would have been interesting if information on sleep on weekdays and weekends was 
collected separately, but since the questionnaire has a 1 -week recall, this data represents sleep on an average day. Seasonal differences and translation could have possibly biased the results in the cultural comparison.

\section{Conclusion}

The prevalence of sleep problems in healthy Dutch schoolaged children is comparable to percentages found in other populations, but specific sleep problems vary between different cultures. Cognitions and attitudes toward what is considered good or normal sleep seem to play an important role in (proxy and self) appraisal of sleep and probably partly accounts for cultural differences. In an effort to better understand cultural influences of sleep, further studies assessing cultural similarities and differences, and cognitions and attitudes towards sleep are mandatory. These studies should also include the establishment of culture appropriate norms.

Acknowledgements We would like to thank J.A. Owens for granting permission to translate the children's sleep habits questionnaire and the sleep self-report into Dutch, and X. Liu for enabling the comparison of sleep between several cultures.

Conflict of interest The authors declare that they have no conflict of interest.

Open Access This article is distributed under the terms of the Creative Commons Attribution Noncommercial License which permits any noncommercial use, distribution, and reproduction in any medium, provided the original author(s) and source are credited.

\section{References}

1. Ali NJ, Pitson DJ, Stradling JR (1993) Snoring, sleep disturbance, and behaviour in 4-5 year olds. Arch Dis Child 68:360-366

2. Anders T, Eiben L (1997) Pediatric sleep disorders: a review of the past 10 years. J Am Acad Child Adolesc Psychiatry 36:9-20

3. Archbold K, Pituch K, Panahi P, Chervin R (2002) Symptoms of sleep disturbances among children at two general pediatric clinics. J Pediatr 140:97-102

4. Bloom B, Owens J, McGuinn M et al (2002) Sleep and its relationship to pain, dysfunction, and disease activity in juvenile rheumatoid arthritis. J Rheumatol 29:169-173

5. Boergers J, Hart C, Owens J et al (2007) Child sleep disorders: associations with parental sleep duration and daytime sleepiness. $\mathrm{J}$ Fam Psychol 21:88-94

6. Couturier J, Speechley K, Steele M et al (2005) Parental perception of sleep problems in children of normal intelligence with pervasive developmental disorders: prevalence, severity, and pattern. J Am Acad Child Adolesc Psychiatry 44:815-822

7. Dahl RE (1996) The impact of inadequate sleep on children's daytime cognitive function. Semin Pediatr Neurol 3:44-50

8. Goodlin-Jones B, Sitnick S, Tang K et al (2008) The children's sleep habits questionnaire in toddlers and preschool children. $\mathrm{J}$ Dev Behav Pediatr 29:82-88

9. Gregory AM, Caspi A, Eley TC et al (2005) Prospective longitudinal associations between persistent sleep problems in childhood and anxiety and depression disorders in adulthood. J Abnorm Child Psychol 33:157-163

10. Gregory AM, Rijsdijk FV, Dahl RE et al (2006) Associations between sleep problems, anxiety, and depression in twins at 8 years of age. Pediatrics 118:1124-1132

11. Groves RM (2006) Nonresponse rates and nonresponse bias in household surveys. Public Opin Quart 70:646-675

12. Janse AJ, Gemke RJBJ, Uiterwaal CS et al (2004) Quality of life; patients and doctors don't always agree: a meta analysis. J Clin Epidemiol 57:661

13. Jenni O, O'Connor B (2005) Children's sleep: an interplay between culture and biology. Pediatrics 115:204-216

14. Latz S, Wolf A, Lozoff B (1999) Cosleeping in context: sleep practices and problems in young children in Japan and the United States. Arch Pediatr Adolesc Med 153:339-346

15. Lavigne JV, Arend R, Rosenbaum D et al (1999) Sleep and behavior problems among preschoolers. J Dev Behav Pediatr 20:164-169

16. Liu X, Hubbard J, Fabes R, Adam J (2006) Sleep disturbances and correlates of children with autism spectrum disorders. Child Psychiatry Hum Dev 37:179-191

17. Liu X, Liu L, Owens JA, Kaplan DL (2005) Sleep patterns and sleep problems among schoolchildren in the United States and China. Pediatrics 115:241-249

18. Liu X, Liu L, Wang R (2003) Bed sharing, sleep habits, and sleep problems among Chinese school-aged children. Sleep 26:839-844

19. Long A, Krishnamurthy V, Palermo T (2008) Sleep disturbances in school-age children with chronic pain. J Pediatr Psychol 33:258-268

20. Montgomery-Downs H, Jones V, Molfese V, Gozal D (2003) Snoring in preschoolers: associations with sleepiness, ethnicity, and learning. Clin Pediatr (Phila) 42:719-726

21. Owens J (2005) Introduction: culture and sleep in children. Pediatrics 115:201-203

22. Owens J, Maxim R, Nobile C et al (2000) Parental and self-report of sleep in children with attention-deficit/hyperactivity disorder. Arch Pediatr Adolesc Med 154:549-555

23. Owens JA, Spirito A, McGuinn M, Nobile C (2000) Sleep habits and sleep disturbance in elementary school-aged children. J Dev Behav Pediatr 21:27-36

24. Owens JA, Spirito A, Meenan RF (2000) The children's sleep habits questionnaire (CSHQ) psychometric properties of a survey instrument for school-aged children. Sleep 23:1043-1051

25. Russo P, Bruni O, Lucidi F et al (2007) Sleep habits and circadian preference in Italian children and adolescents. J Sleep Res 16:163-169

26. Sadeh A (1996) Evaluating night wakings in sleep-disturbed infants: a methodological study of parental reports and actigraphy. Sleep 19:757-762

27. Sadeh A, Gruber R, Raviv A (2002) Sleep, neurobehavioral functioning, and behavior problems in school-age children. Child Dev 73:405-417

28. Sadeh A, Sivan Y (2009) Clinical practice: sleep problems during infancy. Eur J Pediatr 168:1159-1164

29. Super CM, Harkness S, Van Tijen N et al (1996) The three R's of Dutch childrearing and the socialization of infant arousal. In: Harkness S, Super CM (eds) Parents' cultural belief systems: their origins, expressions, and consequenses. Guilford, New York

30. Theunissen NC, Vogels TG, Koopman HM et al (1998) The proxy problem: child report versus parent report in health-related quality of life research. Qual Life Res 7:387-397

31. Tikotzky L, Sadeh A (2001) Sleep patterns and sleep disruptions in kindergarten children. J Clin Child Psychol 30:581-591

32. Wiggs L, Montgomery P, Stores G (2005) Actigraphic and parent reports of sleep patterns and sleep disorders in children with subtypes of attention-deficit hyperactivity disorder. Sleep 28: $1437-1445$ 